\title{
Conversion from HST ACS and STIS auroral counts into brightness, precipitated power, and radiated power for $\mathrm{H}_{2}$ giant planets
}

\author{
J. Gustin, ${ }^{1}$ B. Bonfond, ${ }^{1}$ D. Grodent, ${ }^{1}$ and J.-C. Gérard ${ }^{1}$ \\ Received 9 February 2012; revised 8 June 2012; accepted 8 June 2012; published 24 July 2012.
}

[1] The STIS and ACS instruments onboard HST are widely used to study the giant planet's aurora. Several assumptions have to be made to convert the instrumental counts into meaningful physical values (type and bandwidth of the filters, definition of the physical units, etc...), but these may significantly differ from one author to another, which makes it difficult to compare the auroral characteristics published in different studies. We present a method to convert the counts obtained in representative ACS and STIS imaging modes/filters used by the auroral scientific community to brightness, precipitated power and radiated power in the ultraviolet (700-1800 $\AA$ ). Since hydrocarbon absorption may considerably affect the observed auroral emission, the conversion factors are determined for several attenuation levels. Several properties of the auroral emission have been determined: the fraction of the $\mathrm{H}_{2}$ emission shortward and longward of the HLy- $\alpha$ line is $50.3 \%$ and $49.7 \%$ respectively, the contribution of HLy- $\alpha$ to the total unabsorbed auroral signal has been set to $9.1 \%$ and an input of $1 \mathrm{~mW} \mathrm{~m}^{-2}$ produces $10 \mathrm{kR}^{\circ} \mathrm{H}_{2}$ in the Lyman and Werner bands. A first application sets the order of magnitude of Saturn's auroral characteristics in the total UV bandwidth to a brightness of $10 \mathrm{kR}$ and an emitted power of $\sim 2.8 \mathrm{GW}$. A second application uses published brightnesses of Europa's footprint to determine the current density associated with the Europa auroral spot: 0.21 and $0.045 \mu \mathrm{A} \mathrm{m}^{-2}$ assuming no hydrocarbon absorption and a color ratio of 2, respectively. Factors to extend the brightnesses observed with Cassini-UVIS to total $\mathrm{H}_{2}$ UV brightnesses are also provided.

Citation: Gustin, J., B. Bonfond, D. Grodent, and J.-C. Gérard (2012), Conversion from HST ACS and STIS auroral counts into brightness, precipitated power, and radiated power for $\mathrm{H}_{2}$ giant planets, J. Geophys. Res., 117, A07316, doi:10.1029/2012JA017607.

\section{Introduction}

[2] The ultraviolet (UV) aurora of the giant planets were first observed with the UV spectrometers (UVS) onboard the Voyager 1 and 2 spacecraft in the late seventies-early eighties [Broadfoot et al., 1979, 1981; Sandel and Broadfoot, 1981]. Since then, significant scientific advances have been made with improved observational platforms. In particular, the Space Telescope Imaging Spectrograph (STIS) and the Advanced Camera for Survey (ACS) onboard the Hubble Space Telescope (HST) are leading instruments participating in the progress of planetary auroral science. The purpose of this study is to establish the conversion factors from the counts detected in ACS and STIS images to physical quantities needed to characterize the auroras and useful to infer the processes leading to these emissions.

\footnotetext{
${ }^{1}$ Laboratoire de Physique Atmosphérique et Planétaire, Université de Liège, Liège, Belgium.

Corresponding author: J. Gustin, Laboratoire de Physique Atmosphérique et Planétaire, Université de Liège, Allée du 6 août, 17, Liège BE-4000, Belgium. (j.gustin@ulg.ac.be)

(C2012. American Geophysical Union. All Rights Reserved. 0148-0227/12/2012JA017607
}

[3] The giant planets auroras are produced by the excitation of $\mathrm{H}_{2}$ molecules by electrons precipitating into their atmosphere. The UV emissions are the result of the excited molecules going down to the ground electronic state. They instantaneously reflect the intensity and morphology of the electron precipitation. The characteristics of the aurora and the physical processes inducing them are thus mainly examined through the study of three main variables: the observed brightness, expressed in kilo-Rayleighs $(1 \mathrm{kR}=$ $10^{9} / 4 \pi$ photons $\left.\mathrm{cm}^{-2} \mathrm{sr}^{-1} \mathrm{~s}^{-1}\right)$, the emitted power in $\mathrm{W} \mathrm{m} \mathrm{m}^{-2}$ and the local electron energy input rate in $\mathrm{mW} \mathrm{m}^{-2}$. Various approaches may be used by the different teams to determine these variables (different UV bandwidths, different conversion factors, ...). For the sake of consistency, it is thus important to provide the community with a standard method to determine them. ACS and STIS have a large number of imaging modes and filters, but very few are effectively used in this field of study. We focus here on the main configurations employed for auroral FUV observations: the Solar Blind Channel (SBC) with the F115LP or F125LP filters for ACS, and the FUV MAMA (MultiAnode Microchannel Array) detector with the CLEAR (25MAMA) and Strontium Fluoride (F25SrF2) filters for STIS. The conversions to be determined are valid for pipelined files corrected for several 
effects, such as dark count subtraction, flat-field, geometric and photometric distortions, i.e., x2d.fits and drz.fits / flt. fits data files for STIS and ACS instruments, respectively. Factors to convert the brightnesses obtained with the Cassini UltraViolet Imaging Spectrograph (UVIS) spectrometers to total unabsorbed $\mathrm{H}_{2}$ brightnesses are also provided, which will allow to directly compare results obtained with STIS, ACS and UVIS, the three most frequently used UV instruments in planetary sciences.

\section{Auroral Characteristics}

[4] The auroral emissions on the giant planets in the UV (700-1800 $\AA$ ) stem from the emission of atomic $\mathrm{H}$ lines from the Lyman series and $\mathrm{H}_{2}$ vibronic lines + continuum from the $\mathrm{B}^{1} \sum_{\mathrm{g}}^{+} \rightarrow \mathrm{X}^{1} \sum_{\mathrm{g}}^{+}, \mathrm{C}^{1} \prod_{\mathrm{u}} \rightarrow \mathrm{X}^{1} \sum_{\mathrm{g}}^{+}, \mathrm{B}^{\prime 1} \sum_{\mathrm{u}}^{+} \rightarrow \mathrm{X}^{1} \sum_{\mathrm{g}}^{+}$, $\mathrm{D}^{1} \prod_{\mathrm{u}} \rightarrow \mathrm{X}^{1} \sum_{\mathrm{g}}^{+}, \mathrm{B}^{\prime \prime 1} \sum_{\mathrm{u}}^{+} \rightarrow \mathrm{X}^{1} \sum_{\mathrm{g}}^{+}$and $\mathrm{D}^{\prime 1} \prod_{\mathrm{u}} \rightarrow \mathrm{X}^{1} \sum_{\mathrm{g}}^{+}$ system bands. These bands are produced by the excitation of $\mathrm{H}_{2}$ ground-state molecules by electrons of magnetospheric origin precipitating into the atmosphere. In the FUV (1200$1800 \AA$ ), the signal is dominated by H Lyman- $\alpha$ (Ly- $\alpha)$ and the Lyman $(\mathrm{B} \rightarrow \mathrm{X})$ and Werner $(\mathrm{C} \rightarrow \mathrm{X})$ bands. An ionospheric hydrocarbon layer interacts with the aurora and attenuates the emission in specific wavelength ranges. Three hydrocarbons have a significant optical depth in the UV: methane $\left(\mathrm{CH}_{4}\right)$, ethane $\left(\mathrm{C}_{2} \mathrm{H}_{6}\right)$ and acetylene $\left(\mathrm{C}_{2} \mathrm{H}_{2}\right)$, with a clear domination of $\mathrm{CH}_{4}$. Methane attenuates the $\mathrm{H}_{2}$ emission shortward of $1350 \AA$, leaving the emission longward of $1350 \AA$ unattenuated. This absorption is measured by the color ratio $\mathrm{CR}=\mathrm{I}(1550-1620 \AA) / \mathrm{I}(1230 / 1300 \AA)$ with $\mathrm{I}$ the intensity in photon units [Yung et al., 1982]. It relates the attenuation of the auroral emission to the amount of hydrocarbons overlying the emission layer. It is thus an indicator of the relative penetration depth of the primary electrons into the hydrocarbon layer and can be related to their incident energy. The conversion factors derived hereafter consider that all the auroral emission is due to electron precipitation, as demonstrated by the study by Trafton et al. [1998] and Liu and Schultz [2000]. By contrast, the X-ray aurora is principally due to sulfur and oxygen ions precipitation [Waite et al., 1994; Kharchenko et al., 2006].

[5] Several spectroscopic studies show that the CR, which is $\sim 1.1$ for an unattenuated emission, usually varies from 1.4 to 10 for Jupiter [Gérard et al., 2002; Gustin et al., 2004]. Two exceptional events where observed with STIS in 1999, with CRs of 18.5 and 45.5 [Gustin et al., 2006]. It is difficult to establish a standard value, as the CR may vary significantly with time and location, but 2.5 is generally used as a typical number in image studies where the CR cannot be directly determined. This value corresponds to the precipitation of electrons of $\sim 75 \mathrm{keV}$ [Gustin et al., 2004]. In the case of Saturn, signatures of hydrocarbon absorption are weaker and rarer. A current study of several hundreds of auroral spectra obtained with the Cassini UltraViolet Imaging Spectrograph (UVIS) reveals that $\sim 15 \%$ of them exhibit a weak signature of methane absorption, while less than $10 \%$ show a significant absorption with CR between $\sim 1.4$ and $\sim 2.7$, with only two cases showing a remarkable CR of $\sim 4$. In other words, these spectroscopic studies demonstrate that the auroral emission is produced deep inside the layer of hydrocarbons in the case of Jupiter, and close to the homopause level in the case of Saturn. It should be noted that we assume here the simple case where the aurora consists of an emitting layer surrounded or overlaid by an absorption layer. This means that the amount of absorption depends on the angle between the local vertical of the region observed and the observer, and that the CR depends on the viewing geometry.

[6] The emission from HLy- $\alpha$ is an important matter, as images obtained without appropriate filters integrate this component along with the $\mathrm{H}_{2}$ bands. In such case, it is useful to know how to isolate this contribution from the observation to get pure $\mathrm{H}_{2}$ emission. Three mechanisms contribute to HLy- $\alpha$ : scattering of Solar HLy- $\alpha$, direct excitation of $\mathrm{H}$ atoms and dissociative excitation of $\mathrm{H}_{2}$ by electron impact. A first step is the determination of the HLy- $\alpha$ contribution to the total $\mathrm{H}_{2} \mathrm{UV}$ emission. This can be done by fitting a synthetic $\mathrm{H}_{2}$ spectrum obtained from impact of $100 \mathrm{eV}$ electrons with a gas at $300 \mathrm{~K}$ [Dols et al., 2000; Gustin et al., 2004] to various observations. This $\mathrm{H}_{2}$ spectrum reproduces well a typical unabsorbed auroral spectrum. The fitting regression includes a single line at $1215.65 \AA$ simulating HLy- $\alpha$, and absorption by methane, ethane and acetylene. The model intensity of $\mathrm{H}_{2}, \mathrm{HLy}-\alpha$ and the amount of hydrocarbon vary independently until a best fit is obtained. The $\mathrm{H}_{2}$ and HLy- $\alpha$ unabsorbed emissions are then determined and allow to establish the contribution of each component. Since different instruments are active in different UV bandpasses, a convenient way to uniformize the results is to extrapolate the intensities obtained in a limited bandpass to the whole $\mathrm{H}_{2} \mathrm{UV}$ bandwidth, from 700 to $1800 \AA$, which is easily done with synthetic $\mathrm{H}_{2}$ spectra.

[7] The first observation examined is a STIS Jovian auroral spectrum obtained on 14 November 2000, with the G140L grating and CLEAR filter, known to have a standard CR of 2.5 [Gustin et al., 2004, Table 1]. The best fit shows that HLy- $\alpha$ is $12.6 \%$ of the total unabsorbed $\mathrm{H}_{2}$, i.e., HLy- $\alpha$ contributes $11.1 \%$ of the total $\mathrm{H}_{2}+\mathrm{HLy}-\alpha$ emission.

[8] We then examined several hundreds of Saturn auroral spectra obtained with UVIS and determined the $\mathrm{H}_{2}$ and HLy- $\alpha$ unabsorbed brightnesses. The $\mathrm{H}_{2}$ - HLy- $\alpha$ observed relationship was fitted with a linear regression (J. Gustin et al., manuscript in preparation, 2012) and it was found that HLy- $\alpha$ corresponds to $8.4 \%$ of the total unabsorbed $\mathrm{H}_{2}$ emission, thus contributing $7.7 \%$ of the total auroral emission in 700-1800 $\AA$.

[9] We also fitted the laboratory $\mathrm{H}_{2}$ spectrum at $300 \mathrm{~K}$ and $100 \mathrm{eV}$ described by Liu et al. [1998] and Dziczek et al. [2000] with our regression procedure. In this case, the HLy- $\alpha$ line represents $9.5 \%$ of the total $\mathrm{H}_{2}$ and corresponds to $8.6 \%$ of the total UV emission.

[10] The proportion of HLy- $\alpha$ can also be estimated theoretically by using the excitation cross-sections of HLy- $\alpha$ and $\mathrm{H}_{2}$ by electron impact. Simulations with the two stream model described by Grodent et al. [2001] show that HLy- $\alpha$ is $\sim 13 \%$ of the total $\mathrm{H}_{2}$ unabsorbed emission and represents $11.9 \%$ of the total auroral UV emission. In addition, this model reveals that the production of HLy- $\alpha$ by electron impact is primarily due to dissociative excitation of $\mathrm{H}_{2}$, which contributes $\sim 99 \%$. These results indicate that the fraction of HLy- $\alpha$ to the total $\mathrm{H}_{2}$ varies from $\sim 8$ to $13 \%$, depending on the observation condition or input parameters used in theoretical models. We recommend to set to $10 \%$ the standard value of the $\mathrm{HLy}-\alpha / \mathrm{H}_{2}$ ratio, implying that 


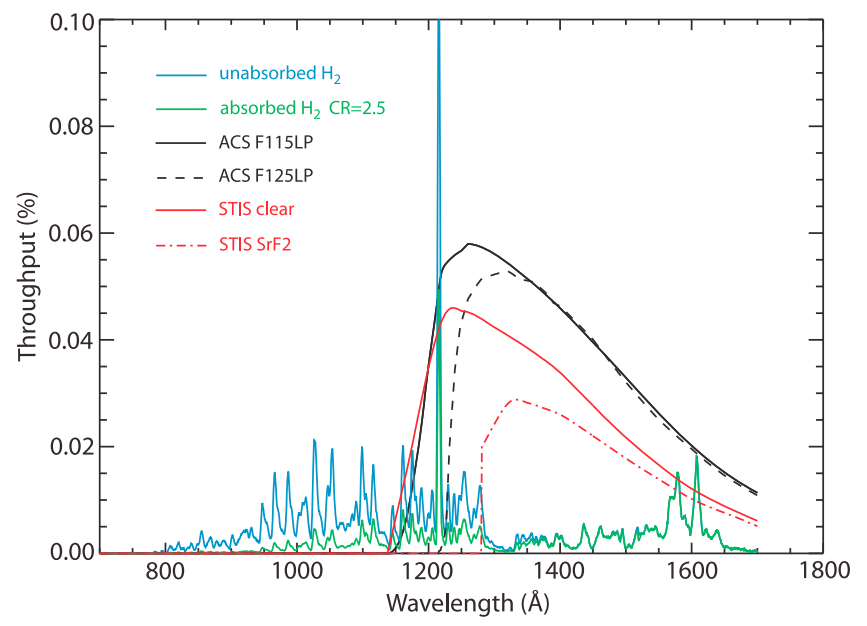

Figure 1. Total system throughput for ACS F115LP, F125LP and STIS CLEAR, SrF2 filters. A H2 laboratory spectrum shown in blue is used to simulate the auroral unabsorbed emission. An example of $\mathrm{H} 2$ emission affected by the hydrocarbon layer is shown green, for a CR of 2.5. The $\mathrm{H} 2$ spectra are scaled to fit the window.

HLy- $\alpha$ contributes $9.1 \%$ of the total unabsorbed UV auroral emission.

\section{ACS and STIS Throughputs and Conversions}

[11] The instrument throughput is defined as the number of photons counted on the detector divided by the incoming flux of photons intersecting the surface area of the telescope primary mirror. It depends on the individual components in the optical path of the telescope and scientific instruments (reflection efficiency of the telescope optics and internal mirrors of the instrument), the transmission of the filters and the quantum efficiency of the detectors. In the case of HST, the first element is the OTA (optical Telescope Assembly), which is composed of the primary mirror capturing light from observed objects and a secondary mirror which redirects lights from the primary mirror to the science instruments. Before 1993, a corrective optics which compensated for the imperfect shape of the primary mirror (COSTAR) was included in OTA. It became unnecessary after 1993, as the optics of all later science instruments included built-in corrective optics. In the following, the transmission specific to the STIS or ACS optics and detectors are taken into account in order to derive the total system wavelength-dependent throughput. The ACS and STIS absolute calibration is known with an uncertainty of about $10 \%$. The physical quantities deduced from the conversion factors are thus estimated with an error bar of about $10 \%$.

\subsection{ACS}

[12] ACS was installed onboard HST on March 2002 to replace the Faint Object Camera. It consists of three channels: the Wide Field Channel (WFC) mounted with a chargecoupled device (CCD) sensitive to $\sim 3500$ to $11000 \AA$, the High-Resolution Channel (HRC), also mounted with a CCD detector, sensitive to $\sim 1700$ to $11000 \AA$, and a Solar Blind Channel (SBC) which uses a MAMA detector, sensitive to $\sim 1150$ to $1700 \AA$ spectral window, with a field of view of $34.6^{\prime \prime} \times 30.8^{\prime \prime}$. A complete description of the instrument is found in Maybhate and Armstrong [2010]. The auroral observations are usually acquired with the SBC channel and two filters: the F115LP, which is a $\mathrm{MgF}_{2}$ filter that covers the whole MAMA bandwidth, and the F125 $\mathrm{LP} \mathrm{CaF}_{2}$ filter, which blocks emission from HLy- $\alpha$ and below. The total system throughput of F115LP and F125LP can be obtained online at http://www.stsci.edu/hst/ acs/documents/handbooks/cycle19/c10_ImagingReference38. $\mathrm{html}$ and http://www.stsci.edu/hst/acs/documents/handbooks/ cycle19/c10_ImagingReference $40 . h t m l$, respectively. The total system throughput for these two ACS configurations are shown in Figure 1. The $\mathrm{H}_{2}$ and HLy- $\alpha$ emissions with and without hydrocarbon absorption are also displayed to show the wavelength ranges affected by the filters.

\subsection{STIS}

[13] STIS is a spectro-imager, installed on HST during the second servicing mission in February 1997 [Kimble et al., 1998]. STIS has three $1024 \times 1024$ detector arrays. The first one is a CCD covering the visible and near-infrared spectrum from 2000 to $10300 \AA$ while the other two detectors are MAMAs, each with a $25^{\prime \prime} \times 25^{\prime \prime}$ field of view. One operates in the near-UV between 1600 and $3100 \AA$, the other one covers the FUV between 1150 and $1700 \AA$. For auroral observations, the FUV MAMA is generally used with either the CLEAR filter, which provides high throughput over the whole FUV bandpass, or the 25SRF2 filter which efficiently rejects the HLy- $\alpha$ emission. The corresponding total throughput are available at $\mathrm{http}: / / \mathrm{www} . \mathrm{stsci}$.edu/hst/ stis/documents/handbooks/currentIHB/c14 imref22.html and http://www.stsci.edu/hst/stis/documents/handbooks/currentIHB/ c14 imref29.html. The total system throughput are shown in Figure 1.

\subsection{Conversion Factors}

\subsubsection{Raw Counts to Brightness}

[14] The conversion from count rate detected by the instrument to $\mathrm{kR}$ of unabsorbed auroral emission can be achieved in a two steps process. The first step is the conversion from detected counts to $\mathrm{kR}$. Following Maybhate and Armstrong [2010], the count rate per pixel C due to an extended astronomical source can be expressed as

$$
\mathrm{C}=\mathrm{A} \int \mathrm{I}_{\lambda} \mathrm{Q}_{\lambda} \mathrm{T}_{\lambda} \mathrm{m}_{\mathrm{x}} \mathrm{m}_{\mathrm{y}} \mathrm{d} \lambda
$$

where

$\mathrm{A}$ is the area of the unobstructed $2.4 \mathrm{~m}$ telescope

$I_{\lambda}$ is the surface brightness of the source, in photons second ${ }^{-1} \mathrm{~cm}^{-2} \AA^{-1}$ arcseconds $^{-2}$

$\mathrm{Q}_{\lambda}$ is the instrument sensitivity and $\mathrm{T}_{\lambda}$ is the filter transmission: $\mathrm{Q}_{\lambda} \mathrm{T}_{\lambda}$ thus represents the probability of detecting a count per incident photon

$\mathrm{m}_{\mathrm{x}}$ and $\mathrm{m}_{\mathrm{y}}$ are the plate scale of a pixel along the orthogonal $\mathrm{X}$ and $\mathrm{Y}$ axis. A plate scale of $0.024^{\prime \prime} \times 0.024 "$ and $0.0338^{\prime \prime} \times 0.0301^{\prime \prime}$ is used for STIS-MAMA and ACS-SBC, respectively. Once $\mathrm{I}_{\lambda}$ is derived from (1), a factor $1 \times 10^{9} / 4 \pi$ must be applied to convert the counts into $\mathrm{kR}$.

[15] The observed brightness so obtained represents the auroral emission in the bandpass of the filter, modified by the 

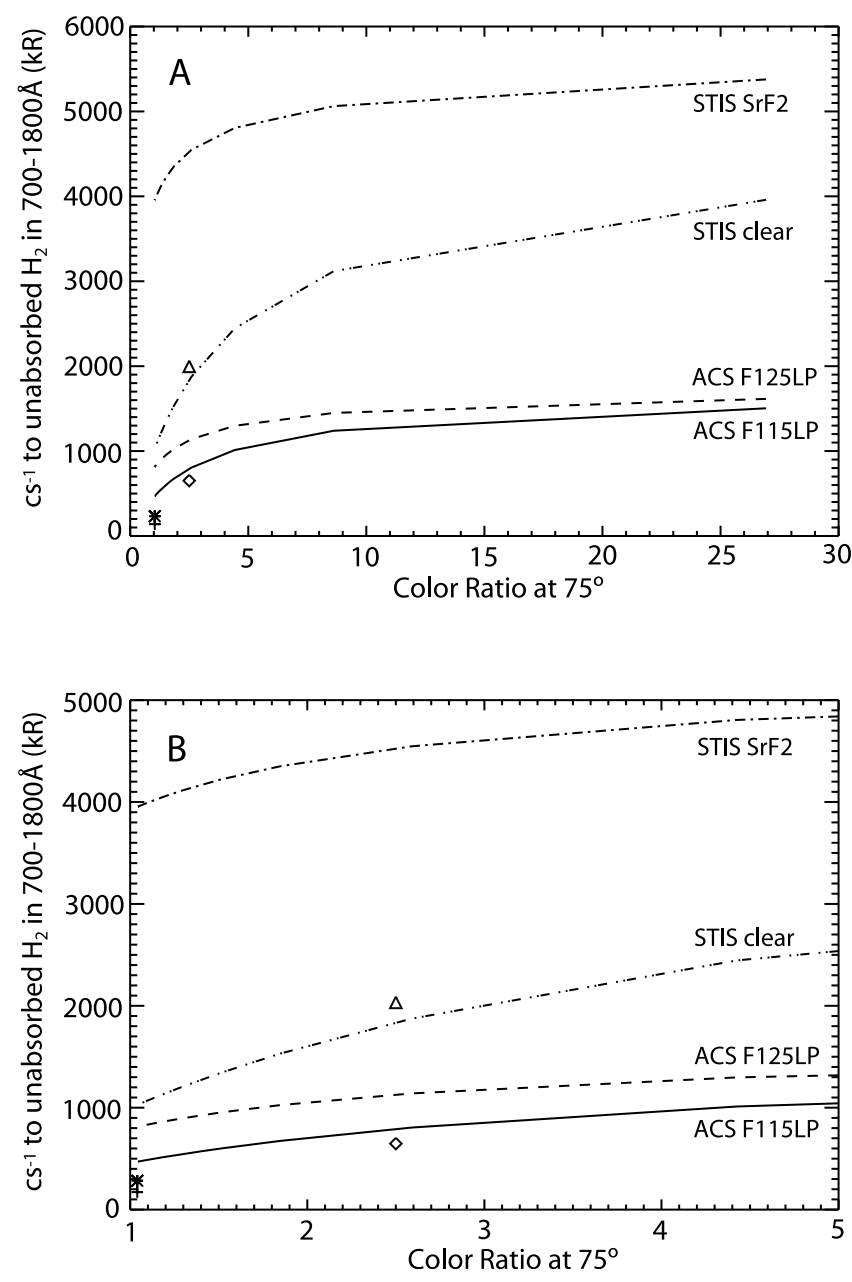

Figure 2. (a) Factors used to convert the count rate from a $\times 2 \mathrm{~d}$ or flt/drz file to $\mathrm{kR}$ of unabsorbed $\mathrm{H}_{2}$ in the 700 $1800 \AA$ bandwidth, including all the Rydberg states. The cross, star, diamond and triangle represent the conversion factor used in previous publications for ACS F115LP, ACS F125LP, STIS CLEAR and STIS SrF2 respectively. (b) Zoom on Figure 2a for low values of the CR.

hydrocarbon absorption. The following method has been employed to derive the intrinsic unabsorbed $\mathrm{H}_{2}$ emission in the 700-1800 $\AA$ bandwidth. As previously stated, absorption by hydrocarbon is not constant but may vary with time and location. It is thus important to determine the conversion factors for different values of the CR. The $\mathrm{H}_{2}$ laboratory spectrum from Dziczek et al. [2000] is used to simulate the auroral unabsorbed emission, on which absorption by $\mathrm{CH}_{4}$, $\mathrm{C}_{2} \mathrm{H}_{2}$ and $\mathrm{C}_{2} \mathrm{H}_{6}$ is applied. In the case of Jupiter, the attenuation of the $\mathrm{H}_{2}$ emission is principally caused by $\mathrm{CH}_{4}$, with small contributions from $\mathrm{C}_{2} \mathrm{H}_{2}$ and $\mathrm{C}_{2} \mathrm{H}_{6}$. We simulate the Jovian atmosphere by using the atmospheric model of Moses et al. [2005] from which we have sampled the mixing ratios of the 3 hydrocarbons for altitudes between 290 to $445 \mathrm{~km}$ in bins of $10 \mathrm{~km}$. The optical depth is calculated for each bin and applied to the laboratory $\mathrm{H}_{2}$ spectrum. The resulting absorbed spectra reproduces the auroral absorbed UV emission, with CR from 1.1 to 27 . In this simple model, the hydrocarbons are modeled as an absorbing layer above the auroral emission layer, with an absorption proportional to the secant of the viewing angle, which depends on the specific observation considered. An angle of $75^{\circ}$ has been chosen to simulate a typical viewing angle between the local vertical and the pointing vector between HST and the target. The different filters are then applied to the absorbed spectrum. The counts per second to $\mathrm{kR}$ conversion factors are given by the ratio between the filtered absorbed spectrum and the $\mathrm{H}_{2}$ laboratory spectrum, from which the HLy- $\alpha$ component was removed. The dependence of the conversion factors with absorption is displayed in Figure 2 and Table 1. The brightness obtained corresponds to the auroral $\mathrm{H}_{2}$ emission in the 700-1800 $\AA$ bandwidth which includes transitions from $\mathrm{B}, \mathrm{C}, \mathrm{B}^{\prime}, \mathrm{D}, \mathrm{B}^{\prime \prime}, \mathrm{B}^{\prime}$ to $\mathrm{X}$ electronic ground state. The cross, star, diamond and triangle symbols represent the factors used in previous studies [e.g., Grodent et al., 2006], which assumed a CR of 2.5 for STIS and no absorption for ACS. A $\mathrm{CR}$ of 2.5 can be considered as a possible standard value to be adopted in Jovian auroral studies, but Figure 2 allows one to choose another $\mathrm{CR}$ for conversion. The earlier factors considered emission in the 1220-1800 A window only with various absorptions, which explains why they are about twice smaller than the new numbers [e.g., Grodent et al., 2006; Radioti et al., 2008; Bonfond et al., 2011]. Indeed, the synthetic unabsorbed $\mathrm{H}_{2}$ spectrum reveals that the fraction below (EUV) and beyond (FUV) HLy- $\alpha$ is 50.3 and $49.7 \%$, respectively. It also shows that the Lyman (B to X transitions) and Werner ( $\mathrm{C}$ to $\mathrm{X}$ transitions) bands contribute $90.4 \%$ of the total $\mathrm{H}_{2}$ emission. It should be noted that these CR-dependent factors are also applicable to the Saturn aurora: the hydrocarbon absorption, when present, is very weak, and can be simulated by the high altitude section of the Moses et al. [2005] model, where ethane and acetylene are negligible. Assuming that the absorption is mainly due to methane, the shape of the emergent auroral spectrum only depends on the total hydrocarbon column traversed by the photons. It is thus independent of the hydrocarbon density profile, i.e., independent of the planet or atmospheric model considered. Although the auroral emission is mostly affected by $\mathrm{CH}_{4}$, we use the model by Moses et al. [2005] to establish the amount of $\mathrm{C}_{2} \mathrm{H}_{2}$ and $\mathrm{C}_{2} \mathrm{H}_{6}$ with respect to $\mathrm{CH}_{4}$, in order to calculate the color ratio with the most possible precision for the higher CR values, where acetylene and ethylene may show their absorption signature. Though this model is appropriate to low latitudes atmosphere, we assume it gives a reasonable estimate of the $\mathrm{C}_{2} \mathrm{H}_{2}$ and $\mathrm{C}_{2} \mathrm{H}_{6}$ mixing ratio at high latitudes.

\subsubsection{Raw Counts to Precipitated Power}

[16] The second physical number to determine is the total precipitating power, that is, the total energy per second carried by the electrons precipitating into the atmosphere. This has been determined by several authors using a continuous slowing down approximation [Gérard and Singh, 1982], a two-stream approximation [Waite et al., 1983; Grodent et al., 2001] and a Monte-Carlo code described by Shematovich et al. [1994] for the Earth's atmosphere and applied to a $\mathrm{H}_{2}$ atmosphere by Gérard et al. [2009]. Assuming a pure $\mathrm{H}_{2}$ atmosphere, these authors calculated the column production rate in the Lyman and Werner bands for an energy input of $1 \mathrm{~mW}$ per square meter. Values of 10.6, 9.2 and $10.0 \mathrm{kR}$ were obtained by Gérard and Singh [1982], Waite et al. [1983] and Grodent et al. [2001], respectively. These values assume that the primary energy of the electrons exceeds a few 
Table 1. Factor Used to Convert the Observed Counts per Second to $\mathrm{kR}$ of Total Unabsorbed $\mathrm{H}_{2}$ for Different Values of the Absorption

\begin{tabular}{ccccc}
\hline CR & ACS F115LP & ACS F125LP & STIS Clear & STIS SrF2 \\
\hline $1.04^{\mathrm{a}}$ & 469 & 815 & 1027 & 3948 \\
1.10 & 488 & 835 & 1072 & 3994 \\
1.50 & 596 & 950 & 1335 & 4215 \\
2.00 & 701 & 1049 & 1602 & 4391 \\
2.50 & 789 & 1127 & 1833 & 4523 \\
3.00 & 852 & 1176 & 2002 & 4605 \\
3.50 & 908 & 1218 & 2157 & 4675 \\
4.00 & 964 & 1261 & 2313 & 4746 \\
4.50 & 1016 & 1300 & 2458 & 4810 \\
5.00 & 1043 & 1318 & 2538 & 4841 \\
6.00 & 1097 & 1355 & 2698 & 4902 \\
7.00 & 1151 & 1391 & 2859 & 4963 \\
8.00 & 1205 & 1427 & 3019 & 5024 \\
9.00 & 1245 & 1453 & 3137 & 5069 \\
10.00 & 1259 & 1462 & 3183 & 5086 \\
12.00 & 1288 & 1480 & 3274 & 5120 \\
14.00 & 1317 & 1498 & 3366 & 5155 \\
16.00 & 1346 & 1516 & 3458 & 5189 \\
18.00 & 1375 & 1534 & 3550 & 5224 \\
20.00 & 1403 & 1552 & 3641 & 5258 \\
25.00 & 1476 & 1597 & 3871 & 5344 \\
\hline
\end{tabular}

${ }^{\text {a Several }} \mathrm{H}_{2}$ laboratory spectra have been obtained through the years. The laboratory spectrum used here covers the whole 700-1800 $\AA$ bandwidth and has an unabsorbed CR of 1.04 (J. Ajello, personal communication, 2009). This is slightly lower than the $\mathrm{CR}$ obtained from another $\mathrm{H}_{2}$ laboratory spectrum used in Gustin et al. [2004], which only covers the 1140-1700 $\AA$ spectral region and has a CR of 1.10 .

hundreds $\mathrm{eV}$. The Monte-Carlo code was used to determine the dependence of auroral emission with the precipitated energy transported by the electrons. This dependence was found to be small. An isotropic Maxwellian flux of $1 \mathrm{~mW} \mathrm{~m}{ }^{-2}$ with mean energies 1,10 and $20 \mathrm{keV}$ electrons generates 9.5, 9.3 and $9.0 \mathrm{kR}$ of $\mathrm{H}_{2}$ emissions in Lyman and Werner bands, while an isotropic mono-energetic flux with the same energies generates $9.2,9.0$ and $8.8 \mathrm{kR}$ in $\mathrm{B}+\mathrm{C}$, respectively. It is seen that the brightness obtained by the different authors with different methods agree well with each other, and we chose a standard value of $10 \mathrm{kR}$ of emission in Lyman and Werner per precipitated $\mathrm{mW} \mathrm{m}^{-2}$, which averages well the brightness obtained by the different authors. The conversion from counts per second to $\mathrm{mW}$ per square meter of precipitated power is easily derived from the conversion factor described in section 3.3.1, as $90.4 \%$ of the total $\mathrm{H}_{2}$ brightness gives the brightness in Lyman + Werner, and $10 \%$ of the latter result gives the precipitated power. Figure 3 presents the conversion factor as a function of the color ratio.

\subsubsection{Raw Counts to Total Emitted Power}

[17] The power radiated by the aurora is an additional important characteristic to consider in the auroral studies. By contrast to the brightness or the precipitated power, the emitted auroral power per unit area depends on the distance from which it is measured, as it decreases with the square of the distance. The conversion from count rate to total power emitted in $4 \pi$ steradians is obtained by determining the power per unit area observed from Earth orbit. The brightness observed by one pixel of the detector is multiplied by $1 \times 10^{9}$ to get the number of photons and the result is multiplied by the mean energy of an auroral photon in $700-1800 \AA$ to obtain the total observed power. The conversion factors, displayed in Table 2 and Figure 4, are calculated for a unit
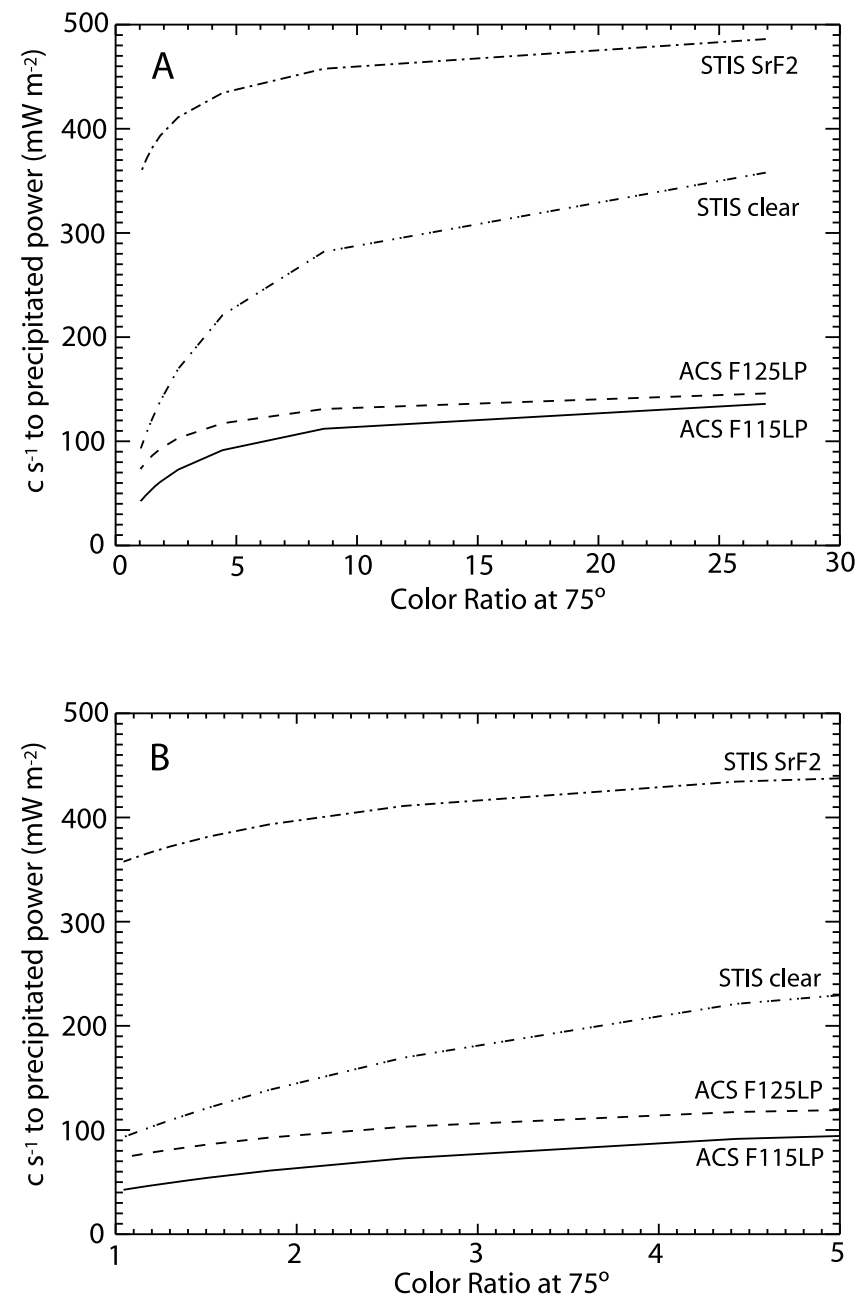

Figure 3. Conversion from count rate to precipitated power.

Table 2. Conversion Factors to Be Multiplied by the Squared HST-Planet Distance (in $\mathrm{km}$ ) to Determine the Total Emitted Power in Watts From Observed Counts per Second

\begin{tabular}{ccccc}
\hline CR & ACS F115LP & ACS F125LP & STIS Clear & STIS SrF2 \\
\hline 1.04 & $1.88 \times 10^{-10}$ & $3.26 \times 10^{-10}$ & $2.32 \times 10^{-10}$ & $8.94 \times 10^{-10}$ \\
1.10 & $1.95 \times 10^{-10}$ & $3.34 \times 10^{-10}$ & $2.43 \times 10^{-10}$ & $9.04 \times 10^{-10}$ \\
1.50 & $2.38 \times 10^{-10}$ & $3.80 \times 10^{-10}$ & $3.02 \times 10^{-10}$ & $9.54 \times 10^{-10}$ \\
2.00 & $2.80 \times 10^{-10}$ & $4.19 \times 10^{-10}$ & $3.63 \times 10^{-10}$ & $9.94 \times 10^{-10}$ \\
2.50 & $3.16 \times 10^{-10}$ & $4.50 \times 10^{-10}$ & $4.15 \times 10^{-10}$ & $1.02 \times 10^{-9}$ \\
3.00 & $3.40 \times 10^{-10}$ & $4.70 \times 10^{-10}$ & $4.53 \times 10^{-10}$ & $1.04 \times 10^{-9}$ \\
3.50 & $3.63 \times 10^{-10}$ & $4.87 \times 10^{-10}$ & $4.88 \times 10^{-10}$ & $1.06 \times 10^{-9}$ \\
4.00 & $3.85 \times 10^{-10}$ & $5.04 \times 10^{-10}$ & $5.24 \times 10^{-10}$ & $1.07 \times 10^{-9}$ \\
4.50 & $4.06 \times 10^{-10}$ & $5.20 \times 10^{-10}$ & $5.56 \times 10^{-10}$ & $1.09 \times 10^{-9}$ \\
5.00 & $4.17 \times 10^{-10}$ & $5.27 \times 10^{-10}$ & $5.74 \times 10^{-10}$ & $1.10 \times 10^{-9}$ \\
6.00 & $4.39 \times 10^{-10}$ & $5.42 \times 10^{-10}$ & $6.11 \times 10^{-10}$ & $1.11 \times 10^{-9}$ \\
7.00 & $4.60 \times 10^{-10}$ & $5.56 \times 10^{-10}$ & $6.47 \times 10^{-10}$ & $1.12 \times 10^{-9}$ \\
8.00 & $4.82 \times 10^{-10}$ & $5.70 \times 10^{-10}$ & $6.83 \times 10^{-10}$ & $1.14 \times 10^{-9}$ \\
9.00 & $4.98 \times 10^{-10}$ & $5.81 \times 10^{-10}$ & $7.10 \times 10^{-10}$ & $1.15 \times 10^{-9}$ \\
10.00 & $5.03 \times 10^{-10}$ & $5.84 \times 10^{-10}$ & $7.20 \times 10^{-10}$ & $1.15 \times 10^{-9}$ \\
12.00 & $5.15 \times 10^{-10}$ & $5.92 \times 10^{-10}$ & $7.41 \times 10^{-10}$ & $1.16 \times 10^{-9}$ \\
14.00 & $5.26 \times 10^{-10}$ & $5.99 \times 10^{-10}$ & $7.62 \times 10^{-10}$ & $1.17 \times 10^{-9}$ \\
16.00 & $5.38 \times 10^{-10}$ & $6.06 \times 10^{-10}$ & $7.83 \times 10^{-10}$ & $1.17 \times 10^{-9}$ \\
18.00 & $5.50 \times 10^{-10}$ & $6.13 \times 10^{-10}$ & $8.03 \times 10^{-10}$ & $1.18 \times 10^{-9}$ \\
20.00 & $5.61 \times 10^{-10}$ & $6.20 \times 10^{-10}$ & $8.24 \times 10^{-10}$ & $1.19 \times 10^{-9}$ \\
25.00 & $5.90 \times 10^{-10}$ & $6.38 \times 10^{-10}$ & $8.76 \times 10^{-10}$ & $1.21 \times 10^{-9}$ \\
\hline & & & &
\end{tabular}



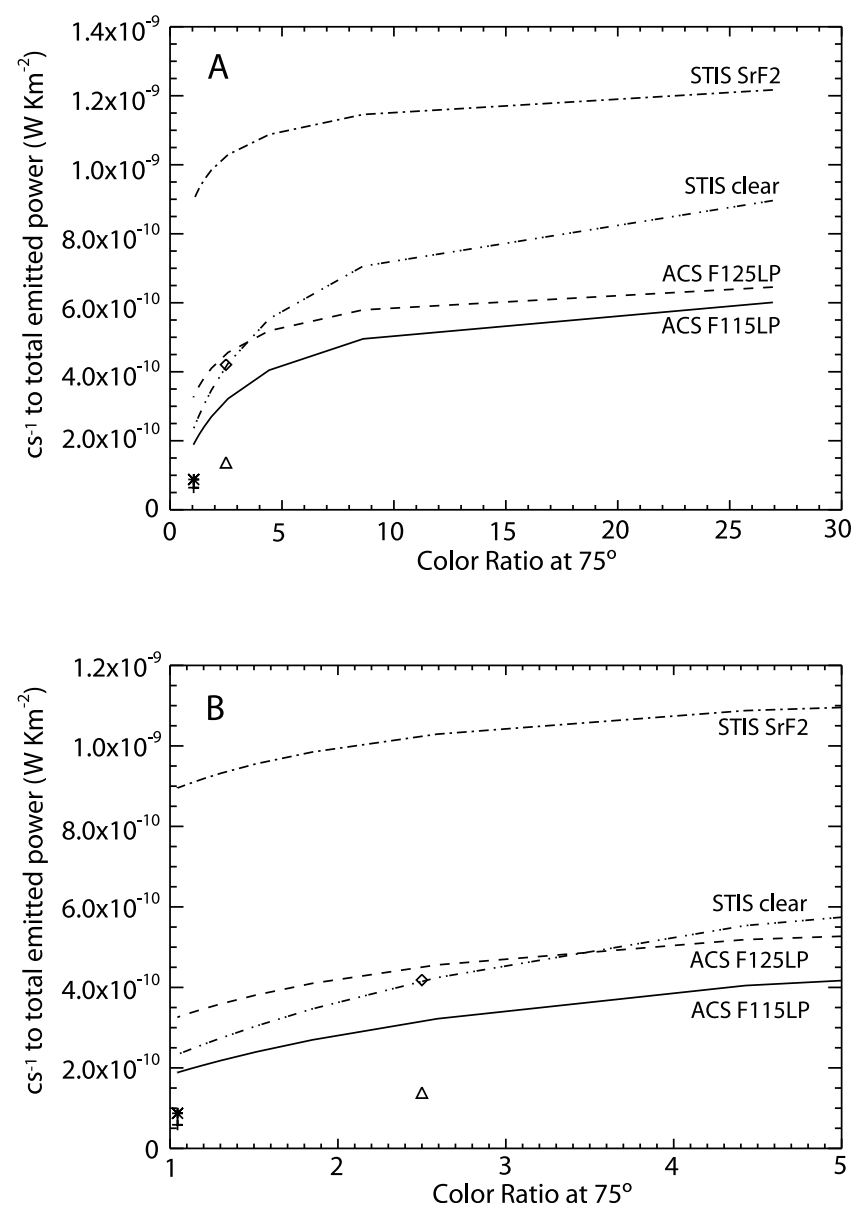

Figure 4. Conversion from count rate to emitted power. The emitted power is observed at Earth's orbit and needs to be corrected for the distance between the emitter and the observer (see section 3.3.4 for more details).

area of $\mathrm{km}^{-2}$, which means that the result must be multiplied by the squared distance between HST and the target at the time of the observation. This information is easily found in the ephemeris.

\subsubsection{Applications}

[18] As a first example, let us consider the $345 \mathrm{~s}$ long timetag observation of Saturn's aurora acquired with the CLEAR filter of STIS on 17 April 2011 at 21:44:38 (Figure 5). The final calibrated and geometrically corrected image has the _x2d.fits extension and is expressed in counts (note that the $\mathrm{x} 2 \mathrm{~d}$ spectroscopic data are expressed in in 'erg $\mathrm{s}^{-1} \mathrm{~cm}^{-2} \AA^{=1} \operatorname{arcsec}^{-2}$ ). We selected 746 pixels in the auroral region near the central meridian longitude (yellow contour in Figure 5), totalizing 7602.67 counts, which gives 10.19 counts pixel $^{-1}$ on average. When considering an auroral region in an image, one must determine a zone on the planet, close to the aurora, which is used to estimate the local background level. Indeed, the observed emission includes photons that do not participate to the intrinsic auroral emission: the planet's airglow, the Hly $\alpha$ and OI lines at $1304 \AA$ coming from Earth's geocorona when HST is in the daylight during the observation and photons from the out-of-band transmission at red wavelengths (red-leak) whose effect may be significant, especially with the ACS camera. All these additional photons contribute to the background and are removed automatically from the auroral emission when the background is subtracted. The background considered here (red contour in Figure 5) has a mean value of 6.97 counts per pixel. The remaining 3.22 counts pixel $^{-1}$ represent the auroral emission, which includes auroral HLy- $\alpha$ and $\mathrm{H}_{2}$ bands in the 1150-1700 $\AA$ bandwidth. To determine the number of $\mathrm{kR}$ of total $\mathrm{H}_{2}$ auroral brightness in [700-1800 ], the counts must be divided by the $345 \mathrm{~s}$ of exposure and then multiplied by the conversion coefficient from Figure 2 and Table 1. For an assumed color ratio of 1.1, this coefficient is 1072 , which gives a mean unabsorbed brightness of $10.0 \mathrm{kR}$. The energy input transported by the precipitating electrons is then $0.9 \mathrm{~mW} \mathrm{~m}^{-2}$, as the emission originating from the B and $\mathrm{C}$ states is $9.04 \mathrm{kR}$. The HLy- $\alpha$ contribution is $9.1 \%$ of the total UV emission, i.e., $0.91 \mathrm{kR}$. The conversion coefficient used to determine the emitted power is the value found in Figure 4 and Table $2\left(2.43 \times 10^{-10}\right.$ in our case $)$, multiplied by the squared HST-Saturn distance at the moment of the observation $\left(1.67066 \mathrm{e} 18 \mathrm{~km}^{2}\right)$. This brings a coefficient of $4.05 \times 10^{8}$, to be multiplied by the total count rate of the auroral region under consideration $\left(6.96 \mathrm{c} \mathrm{s}^{-1}\right)$. The total power emitted by the auroral $\mathrm{H}_{2}$ in $700-1800 \AA$ is then $2.82 \mathrm{GW}$.

[19] In a second example, let us consider the study of Europa's auroral trail made by Grodent et al. [2006]. They analyzed HST-ACS images taken with the F125LP filter, detected the signature of the interaction between Europa and

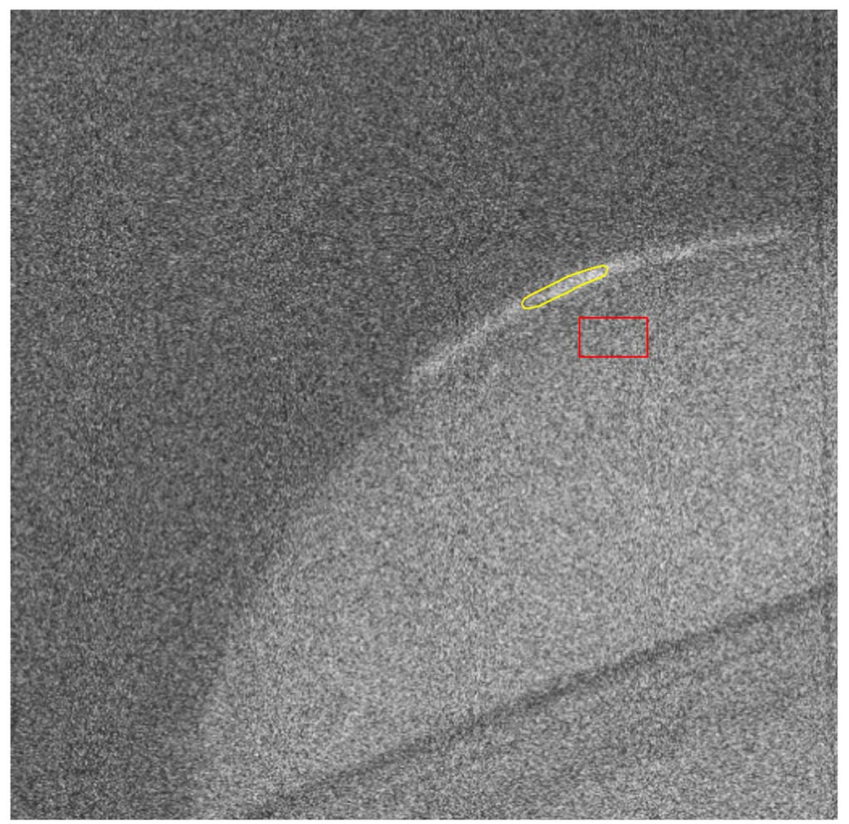

Figure 5. STIS image of Saturn's aurora obtained on 17 April 2011 at 21:44:38. The yellow contour represents the auroral zone examined and the red rectangle is to the background region chosen. The determination of a background near the auroral zone to subtract from the yellow zone is essential, as it contains all the non-auroral emissions: atmospheric airglow, interplanetary Hly $\alpha$, emissions from Earth's geocorona when HST is in the daylight during the observation, photons from the out-of-band transmission at red wavelengths (red-leak). 
the Jovian magnetosphere/ionosphere and established the main characteristics of the emission, such as the brightness and emitted power. They converted the counts per pixel per second to $\mathrm{kR}$ of $\mathrm{H}_{2}$ in 1220-1800 + HLy- $\alpha \AA$, assuming that the observed emission is not affected by hydrocarbon absorption. They obtained a brightness distribution peaking at $\sim 14 \mathrm{kR}$ on the spot with a tail of $\sim 7 \mathrm{kR}$, corresponding to an emitted power of 0.8 and $0.5 \mathrm{GW}$ for the spot and the 1000 by $5000 \mathrm{~km}^{2}$ tail. Using the values expressed in section 3.3.1, if the total $\mathrm{H}_{2}$ in $700-1800 \AA$ is 100 , HLy- $\alpha$ is 10 and $\mathrm{H}_{2}+$ HLy- $\alpha$ in $1220-1800 \AA$ is 59.7 . The published values must be multiplied by $100 / 59.7=1.675$ to get the total $\mathrm{UV}_{2}$ emission, i.e., 23.5 and $11.8 \mathrm{kR}$ for the spot and tail respectively. These figures are multiplied by 0.904 to obtain the emission in Lyman and Werner and multiplied by 0.1 to derive the precipitated power, which gives 2.1 and $1.1 \mathrm{~mW} \mathrm{~m}^{-2}$ for the spot ant tail. The Grodent et al. [2006] analysis assumed that the emission is not affected by hydrocarbon. Taking back Figure 4 in Gérard et al. [2002], this presumes electrons energies less than $\sim 10 \mathrm{keV}$. With $10 \mathrm{keV}$ electrons, the precipitated power corresponds to $1.31 \times 10^{12}$ and $6.6 \times 10^{11}$ electrons per sec per square meter and a current density of 0.21 and $0.11 \mu \mathrm{A} \mathrm{m}^{-2}$ for the spot and tail respectively. As stated in paragraph 3.3.1, the brightness and precipitated power are virtually independent of the atmosphere, as the shape of the $\mathrm{H}_{2}$ emission is controlled by the total amount of absorbing hydrocarbon. On the other hand, the energy of the precipitating electrons and the associated current density do depend on the atmosphere considered. If we make the hypothesis that the signature of Europa's emission is partially absorbed by hydrocarbons, the coefficient used in Grodent et al. [2006] must be adapted. Assuming that the observed emission at the spot is absorbed with a CR of 2 (which is the averaged value measured for the Io footprint), Table 1 shows that the conversion coefficient ratio between unabsorbed and absorbed by $\mathrm{CR}$ of 2 is $815 / 1049=0.78$, which means that only $78 \%$ of the total UV emission emerges from the planet. The total unabsorbed $\mathrm{H}_{2}$ emission at the spot becomes $23.5 / 0.78=30.1 \mathrm{kR}$ and the associated precipitated power is $2.7 \mathrm{~mW} \mathrm{~m}^{-2}$. Gérard et al. [2002, Figure 4] shows that a CR of 2 is attained for electrons of $60 \mathrm{keV}$, i.e., a current density of $0.045 \mu \mathrm{A} \mathrm{m} \mathrm{m}^{-2}$ carrying $2.8 \times 10^{11}$ electrons per second per square meter. The CR-energy dependence published by Gérard et al. [2002] adopt the North Equatorial Belt (NEB) modeled atmosphere described by Gladstone et al. [1996] with a gravity acceleration modified to a latitude of $60^{\circ} \mathrm{N}$. The electron energy/current density values proposed in this example should be adjusted if another atmospheric model is adopted.

\section{UVIS Conversions}

[20] The Cassini-UVIS spectrometers are regularly used to examine Saturn's aurora. When the brightnesses published in these studies only consider the UVIS spectral bandwidth, it is useful to be able to convert these brightnesses to unabsorbed values in the whole $\mathrm{H}_{2} \mathrm{UV}$ window (700-1800 $\AA$ ) in order to compare UVIS and HST derived brightnesses. The UVIS instrument is composed of two spectrographic channels. The EUV UVIS channel covers the spectral range 563-1182 $\AA$. The conversion from this range to unabsorbed $\mathrm{H}_{2} \mathrm{UV}$ emission is 2.06 , i.e., the ratio between the synthetic $\mathrm{H}_{2}$ spectrum in 700-1800 $\AA$ and the $\mathrm{H}_{2}$ laboratory spectrum in 563-1182 $\AA$ described in paragraph 2. The latter was obtained by electron impact and includes the atomic $\mathrm{H}$ lines form the Lyman series in addition to the $\mathrm{H}_{2}$ bands, which makes it ideal to simulate observed auroral emission. The FUV UVIS channel covers the 1115-1912 $\AA$ spectral window, which contains emission from $\mathrm{H}_{2}$ and HLy- $\alpha$. The conversion to unabsorbed $\mathrm{H}_{2} \mathrm{UV}$ emission is given by the ratio between the $\mathrm{H}_{2}$ synthetic spectrum in 700-1800 $\AA$ and the same spectrum in 1115-1912 $\AA$ from which the HLy- $\alpha$ intensity corresponding to $10 \%$ of the total $\mathrm{H}_{2} \mathrm{UV}$ emission has been added (see paragraph 2). The conversion factor obtained is 1.39. These factors are determined assuming that the observed emission is unattenuated by hydrocarbons, which is generally the case at Saturn.

\section{Summary}

[21] This study presents a method to determine the brightness of the UV aurora as well as the energy input transported by the precipitated electrons and the power radiated by the aurora. The accent is put on HST images obtained with STIS using the CLEAR and F25SrF2 filters and with ACS SBC using the F115LP or F125LP filters, commonly used by the auroral scientific community.

[22] Since the observed HST images cannot discriminate the energy of the counted photons, several properties of the auroral signal have been established in order to distinguish the origin of the different auroral emissions and determine their characteristics. By using synthetic, laboratory and observed spectra and several aeronomic models, we make the following statements: 1) the HLy- $\alpha$ line contributes $~ 9 \%$ of the total unabsorbed UV auroral emission, 2) the fraction of unabsorbed $\mathrm{H}_{2}$ emission shortward and longward of HLy- $\alpha$ is $50.3 \%$ and $49.7 \%$ respectively, 3 ) the Lyman and Werner bands contribute $90.4 \%$ of the total unabsorbed $\mathrm{H}_{2}$ emission, 4) the unabsorbed auroral brightness is virtually independent of the mean energy of the precipitated electrons (within $500 \mathrm{eV}-150 \mathrm{keV}$ ) and 5) each input of $1 \mathrm{~mW} \mathrm{~m}^{-2}$ produces $10 \mathrm{kR}$ in the Lyman and Werner bands.

[23] The attenuation of the auroral emission by hydrocarbon is much more important at Jupiter (typical CR of 2.5 ) than at Saturn (no absorption in $80 \%$ of the spectra examined) and it varies with time and location. Accordingly, the conversion from counts to brightness, radiated and precipitated power have been determined for several levels of attenuation.

[24] Since the emergent spectra only depends on the total hydrocarbon column traversed by the auroral photons, the conversion factors are virtually independent of the atmospheric model used. On the other hand, they strongly depend on the assumptions made on the level of absorption.

[25] Two applications show how to apply the conversion factors and auroral characteristics established here. A first example uses the counts detected on a Saturn STIS image and sets for the first time the brightness and emitted power in the total UV bandwidth, with values of $\sim 10 \mathrm{kR}$ and $\sim 2.8 \mathrm{GW}$ respectively, assuming no absorption. A second example uses published brightnesses of Europa's footprint to expand the brightness to the 700-1800 $\AA$ range and derive the associated current density. It is seen that the brightness and 
current density at the Europa spot is $24 \mathrm{kR}$ and $0.21 \mu \mathrm{A} \mathrm{m}^{-2}$ if the emission is supposed unaffected by hydrocarbons, but these values significantly change if absorption is considered (CR of 2), with a brightness of $30 \mathrm{kR}$ and a current density of $0.045 \mu \mathrm{A} \mathrm{m}^{-2}$. Although the case of Jupiter and Saturn are emphasized, the calculations and conversion factors presented in this manuscript are applicable for any giant gas planets dominated by an $\mathrm{H}_{2}$ atmosphere.

[26] Factors to convert the brightnesses obtained with the UVIS spectrometers to total unabsorbed $\mathrm{H}_{2}$ brightnesses are also provided, which allow a straightforward comparison between results obtained with STIS, ACS and UVIS, the three most frequently used UV instruments in planetary sciences. The conversion factors to unabsorbed $\mathrm{H}_{2}$ emission are 2.06 and 1.39 for the UVIS EUV and FUV channels, respectively.

[27] Acknowledgments. The PRODEX program managed by the European Space Agency in collaboration with the Belgian Federal Science Policy Office (BELSPO) provided financial support for this research. This work is supported by the Cassini Project.

[28] Robert Lysak thanks the reviewers for their assistance in evaluating this paper.

\section{References}

Bonfond, B., M. F. Vogt, J.-C. Gérard, D. Grodent, A. Radioti, and V. Coumans (2011), Quasi-periodic polar flares at Jupiter: A signature of pulsed dayside reconnections?, Geophys. Res. Lett., 38, L02104, doi:10.1029/2010GL045981.

Broadfoot, A. L., et al. (1979), Extreme ultraviolet observations from Voyager 1 encounter with Jupiter, Science, 204, 979-982, doi:10.1126/ science.204.4396.979.

Broadfoot, A. L., et al. (1981), Extreme ultraviolet observations from Voyager 1 encounter with Saturn, Science, 212, 206-211, doi:10.1126/ science. 212.4491.206

Dols, V., J.-C. Gérard, J. T. Clarke, J. Gustin, and D. Grodent (2000), Diagnostics of the Jovian aurora deduced from ultraviolet spectroscopy: Model and HST/GHRS observation, Icarus, 147, 251-266, doi:10.1006/ icar.2000.6415.

Dziczek, D., J. M. Ajello, G. K. James, and D. L. Hansen (2000), A study of the cascade contribution to the $\mathrm{H}_{2}$ Lyman band system from electron impact, Phys. Rev. A, 61, 064702, doi:10.1103/PhysRevA.61.064702.

Gérard, J.-C., and V. Singh (1982), A model of energetic electrons and EUV emission in the Jovian and Saturnian atmospheres and implications, J. Geophys. Res., 87, 4525-4532, doi:10.1029/JA087iA06p04525.

Gérard, J.-C., J. Gustin, D. Grodent, P. Delamere, and J. T. Clarke (2002), The excitation of the FUV Io tail on Jupiter: Characterization of the electron precipitation, J. Geophys. Res., 107(A11), 1394, doi:10.1029/ 2002JA009410.

Gérard, J. C., B. Bonfond, J. Gustin, D. Grodent, J. T. Clarke, D. Bisikalo, and V. Shematovich (2009), Altitude of Saturn's aurora and its implications for the characteristic energy of precipitated electrons, Geophys. Res. Lett., 36, L02202, doi:10.1029/2008GL036554.

Gladstone, G. R., M. Allen, and Y. L. Yung (1996), Hydrocarbon photochemistry in the upper atmosphere of Jupiter, Icarus, 119, 1-52, doi:10.1006/icar.1996.0001.
Grodent, D., J. H. Waite Jr., and J. C. Gérard (2001), A self-consistent model of the Jovian auroral thermal structure, J. Geophys. Res., 106, 12,933-12,952, doi:10.1029/2000JA900129.

Grodent, D., J.-C. Gérard, J. Gustin, B. H. Mauk, J. E. P. Connerney, and J. T. Clarke (2006), Europa's FUV auroral tail on Jupiter, Geophys. Res. Lett., 33, L06201, doi:10.1029/2005GL025487.

Gustin, J., et al. (2004), Jovian auroral spectroscopy with FUSE: Analysis of self-absorption and implications for electron precipitation, Icarus, 171, 336-355, doi:10.1016/j.icarus.2004.06.005.

Gustin, J., S. W. H. Cowley, J.-C. Gérard, G. R. Gladstone, D. Grodent, and J. T. Clarke (2006), Characteristics of Jovian morning bright FUV aurora from Hubble Space Telescope/Space Telescope Imaging Spectrograph imaging and spectral observations, J. Geophys. Res., 111, A09220, doi:10.1029/2006JA011730.

Kharchenko, V., A. Dalgarno, D. R. Schultz, and P. C. Stancil (2006), Ion emission spectra in the Jovian X-ray aurora, Geophys. Res. Lett, 33, L11105, doi:10.1029/2006GL026039.

Kimble, R. A., et al. (1998), The on-orbit performance of the Space Telescope Imaging Spectrograph, Astrophys. J., 492, L83-L93, doi:10.1086/ 311102 .

Liu, W., and D. R. Schultz (2000), Ultraviolet emission from oxygen precipitating into Jovian aurora, Astrophys. J., 530, 500-503, doi:10.1086/ 308367.

Liu, X., D. E. Shemansky, S. M. Ahmed, G. K. James, and J. M. Ajello (1998), Electron-impact excitation and emission cross sections of the $\mathrm{H}_{2}$ Lyman and Werner systems, J. Geophys. Res., 103, 26,739-26,758, doi:10.1029/98JA02721.

Maybhate, A., and A. Armstrong (2010), ACS Instrument Handbook, 10th ed., Space Telescope Sci. Inst., Baltimore, Md.

Moses, J. I., T. Fouchet, B. Bézard, G. R. Gladstone, E. Lellouch, and H. Feuchtgruber (2005), Photochemistry and diffusion in Jupiter's stratosphere: Constraints from ISO observations and comparisons with other giant planets, J. Geophys. Res., 110, E08001, doi:10.1029/ 2005JE002411.

Radioti, A. J -C. Gerard, D. Grodent, B. Bonfond, N. Krupp, and J. Woch (2008), Discontinuity in Jupiter's main auroral oval, J. Geophys. Res., 113, A01215, doi:10.1029/2007JA012610.

Sandel, B. R., and A. L. Broadfoot (1981), Morphology of Saturn's aurora, Nature, 292, 679-682, doi:10.1038/292679a0.

Shematovich, V. I., D. V. Bisikalo, and J. C. Gérard (1994), A kinetic model of the formation of the hot oxygen geocorona: 1. Quiet geomagnetic conditions, J. Geophys. Res., 99, 23,217-23,228, doi:10.1029/ 94JA01769.

Trafton, L. M., V. Dols, J.-C. Gérard, J. H. Waite, G. R. Gladstone, and G. Munhoven (1998), HST spectra of the Jovian ultraviolet aurora: Search for heavy ion precipitation, Astrophys. J., 507, 955-967, doi: $10.1086 / 306338$.

Waite, J. H., Jr., T. E. Cravens, J. U. Kozyra, A. F. Nagy, S. K. Atreya, and R. H. Chen (1983), Electron precipitation and related aeronomy of the Jovian thermosphere and ionosphere, J. Geophys. Res., 88, 6143-6163, doi:10.1029/JA088iA08p06143.

Waite, J. H., Jr., F. Bagenal, F. Seward, C. Na, G. R. Gladstone, T. E. Cravens, K. C. Hurley, J. T. Clarke, R. Elsner, and S. A. Stern (1994), ROSAT observations of the Jupiter aurora, J. Geophys. Res., 99(A8), 14,799-14,809, doi:10.1029/94JA01005.

Yung, Y. L., G. R. Gladstone, K. M. Chang, J. M. Ajello, and S. K. Srivastava (1982), $\mathrm{H}_{2}$ fluorescence spectrum from 1200 to $1700 \mathrm{~A}$ by electron impact: Laboratory study and application to jovian aurora, Astrophys. J., 254, L65-L69, doi:10.1086/183757. 